\title{
Ipilimumab-induced autoimmune hypophysitis: a differential for sellar mass lesions
}

\author{
Beverly T Rodrigues ${ }^{1,3}$, Zulfiquer Otty ${ }^{2}$, Kunwarjit Sangla' and Vasant V Shenoy ${ }^{1,3}$ \\ Departments of ${ }^{1}$ Diabetes and Endocrinology ${ }^{2}$ Oncology, The Townsville Hospital, Townsville, \\ Queensland, Australia \\ ${ }^{3}$ School of Medicine and Dentistry, James Cook University, Douglas, Queensland, Australia
}

\author{
Correspondence \\ should be addressed \\ to B T Rodrigues \\ Email \\ beverly.rodrigues@ \\ my.jcu.edu.au
}

\section{Summary}

Autoimmune hypophysitis ( $\mathrm{AH}$ ) has been previously described in a typical demographic population, primarily women in the reproductive age group and perinatal period. The era of immune modulation using anti-cytotoxic T-lymphocyte-associated antigen 4 biological therapy (ipilimumab) against advanced cancers like metastatic melanomas has now resulted in a new form of hypophysitis being increasingly recognised under a spectrum of immune-related adverse events. Drug-related $\mathrm{AH}$ often presents with subtle symptoms and a pituitary mass, with the potential for fatality necessitating wide awareness and a high index of clinical suspicion given that it is usually treatable. We describe below two cases of AH within the last three months at our centre, which were treated with different regimens and produced good endocrine outcomes.

\section{Learning points:}

- $\mathrm{AH}$ is a new and defined clinical entity occurring as a side effect of ipilimumab, which enhances immune-mediated destruction of metastatic melanoma.

- It can present insidiously and have life-threatening complications related to hypocortisolism, hence a high index of clinical suspicion must be exerted by treating physicians, and seems to result in resolution of pituitary masses and variable improvements of pituitary function.

- Clinical improvement, radiological resolution of pituitary masses and variable normalisation of pituitary function are possible with early treatment with high-dose oral or i.v. steroids and hormone replacement therapy, although duration and dosing protocols are unclear at this stage.

- Ipilimumab should continue to be prescribed as treatment for metastatic melanoma; however, close clinical observation of patient's progress must be maintained while they are on this drug.

- Predictive factors for onset of $\mathrm{AH}$ remain unclear and it is imperative that $\mathrm{AH}$ is distinguished from pituitary metastases.

- Further studies are required to determine the safety of continuing therapy with ipilimumab in patients who have developed $\mathrm{AH}$ while on treatment.

\section{Background}

This case series accentuates the importance of a high index of clinical suspicion among cancer patients on ipilimumab therapy. Although not uncommon, autoimmune hypophysitis (AH) is a relatively new clinical entity which, if left untreated, can have potentially lifethreatening complications. Treatment of this condition is available and successful, establishing resolution of clinical symptomology, highlighting the importance of early detection. This case series describes accurate diagnosis 
and treatment options for patients who present with ipilimumab-induced hypophysitis.

\section{Case presentation}

\section{Case 1}

A 64-year-old male presented with a large destructive lesion of the T9 vertebra, due to metastatic melanoma. He was treated with palliative radiotherapy and commenced on $3 \mathrm{mg} / \mathrm{kg}$ dose regimen of ipilimumab, usually administered 3-4 weeks apart in up to four doses. However, following the third dose, he reported extreme fatigue, somnolence, emotional lability, retro-orbital headaches, constipation, anorexia and nausea. Biochemical findings confirmed central hypocortisolism and hypothyroidism (refer Table 1). Magnetic resonance imaging (MRI) of the pituitary revealed a $9 \mathrm{~mm}$ TI hypointense contrast enhancing anterior pituitary mass with marginally increased stalk thickness of $3.3 \mathrm{~mm}$ (Fig. 1A). He did not have evidence of diabetes insipidus.

Given the acute presentation and imaging features suggesting a high likelihood of $\mathrm{AH}$, he was commenced on $75 \mathrm{mg}$ oral prednisolone once daily for 2 weeks, and thyroxine $\left(\mathrm{T}_{4}\right)$ replacement at $75 \mu \mathrm{g}$ daily. He made remarkable symptomatic improvement and the steroids were tapered to $50 \mathrm{mg}$ prednisone for 2 weeks, and then at weekly intervals to 25,10 and $7.5 \mathrm{mg}$. Eight weeks later, he was clinically well with normal pituitary function, with significant improvement on a progress MRI with reduction of the pituitary gland to $5.5 \mathrm{~mm}$, and normal pituitary stalk thickness at $2.1 \mathrm{~mm}$ (Fig. 1B). He is now off $\mathrm{T}_{4}$ and low-dose maintenance hydrocortisone in divided doses (14 mg/day) with a plan to taper further as tolerated. His melanoma disease burden remains stable thus far.

\section{Case 2}

A 65-year-old woman presented with metastatic melanoma in the liver and peritoneum for which she was commenced on $3 \mathrm{mg} / \mathrm{kg}$ of ipilimumab. She initially tolerated the treatment well, but further developed increasing fatigue, weight gain of $6 \mathrm{~kg}$ and bloating, along with elevation in blood glucose levels to $8-13 \mathrm{mmol} / 1$ (144-234 mg/dl) following her third dose. Her blood tests revealed secondary hypothyroidism, normal prolactin levels and central hypogonadism (Table 1). MRI detected a new hypointense heterogeneous mass of the pituitary gland, measuring $12 \mathrm{~mm}$ craniocaudally, and $13 \mathrm{~mm}$ anteroposteriorly, causing slight distortion of the optic chiasm suggesting ipilimumab-induced hypophysitis (Fig. 2A). She was commenced on 3 days of i.v. methylprednisolone at $80 \mathrm{mg}$ once daily as an in-patient, followed by a tapering regimen of high-dose oral prednisolone for 4 weeks starting at $80 \mathrm{mg}$ daily, in addition to $50 \mu \mathrm{g}$ of daily $\mathrm{T}_{4}$.

Four weeks later, she demonstrated marked clinical improvement, with normal thyroid hormone levels (Table 1) and resolution of the pituitary mass on MRI, which now showed a homogeneous enhancement measuring $1 \times 0.3 \times 0.3 \mathrm{~cm}$ with a central stalk completely clear of the optic chiasm (Fig. 2B). Her reproductive hormones were not assayed due to her post-menopausal state. Oral prednisolone was tapered to $10 \mathrm{mg}$ for 2 weeks, followed by $5 \mathrm{mg}$ until further review. Unfortunately, the disease did not respond to ipilimumab and progressed rapidly. She was transferred to the palliative care unit where she ultimately succumbed to her disease. She, however, needed maintenance $\mathrm{T}_{4}$ and prednisone over the period.

\section{Investigation}

Provided separately in table and figure format.

Table 1 Table outlining hormone levels in the patients' pre- and post-high-dose steroid therapy. Values and normal references in SI units and parentheses indicate conventional units.

\begin{tabular}{|c|c|c|c|c|}
\hline & \multicolumn{2}{|c|}{ Case 1} & \multicolumn{2}{|c|}{ Case 2} \\
\hline & Pre & Post & Pre & Post \\
\hline АCTH - 10-50 ng/l (10-50 pg/ml) & $10(10)$ & $21(21)$ & $73(73)$ & $10(10)$ \\
\hline Cortisol - 50-650 nmol/l $(5-25 \mu \mathrm{g} / \mathrm{dl})$ & $62(2.25)$ & $269(9.75)$ & $669(24.25)$ & $813(29.5)$ \\
\hline $\mathrm{TSH}-0.5-5 \mathrm{mlU} / \mathrm{l}$ & 0.08 & 0.6 & 0.04 & 0.8 \\
\hline $\mathrm{FT}_{3}-2.5-6.0 \mathrm{pmol} / \mathrm{l}(162-390 \mathrm{pg} / \mathrm{dl})$ & $2.8(156)$ & $4.8(312)$ & $2.3(149)$ & $2.9(188)$ \\
\hline $\mathrm{FT}_{4}-10-20 \mathrm{pmol} / \mathrm{l}(0.9-2.3 \mathrm{ng} / \mathrm{dl})$ & $6.1(0.47)$ & $11(0.85)$ & $10.2(0.79)$ & $12.9(1.0)$ \\
\hline Prolactin $<500 \mathrm{mIU} / \mathrm{l}(4-23 \mu \mathrm{g} / \mathrm{l})$ & $174(4.0)$ & $266(6.12)$ & $167(3.84)$ & 338 (7.77) \\
\hline $\mathrm{LH}$ & 1.8 & 2.2 & 2 & 1 \\
\hline FSH & 6.6 & 1.5 & 6 & 6 \\
\hline Testosterone & 11 & 15 & & \\
\hline Oestrogen & & & 61 & $<37$ \\
\hline
\end{tabular}


A

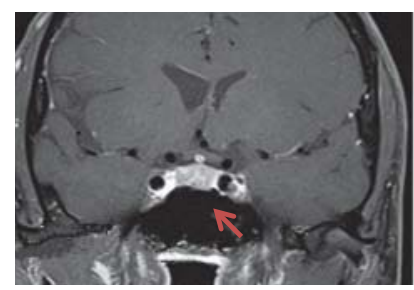

B

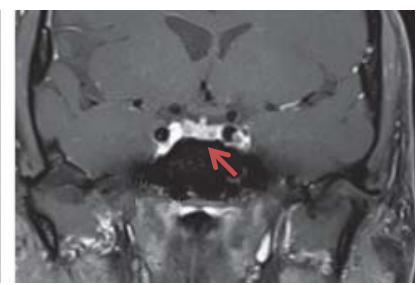

Figure 1

(A) Pre-treatment T1 contrast MRI scan showing a $9 \mathrm{~mm}$ hypointense mass and stalk dimension of $3.3 \mathrm{~mm}$. (B) Post-treatment T1 contrast MRI scan showing reduction of pituitary size to $5.5 \mathrm{~mm}$ and pituitary stalk to $2.1 \mathrm{~mm}$. Arrows indicate pituitary masses.

\section{Treatment}

Treatment provided has been described previously within case description.

\section{Outcome and follow-up}

Case 1 was commenced on high-dose oral prednisolone daily for 2 weeks, and ongoing $\mathrm{T}_{4}$ replacement, on which he improved clinically, allowing for a steroid tapering regimen to be commenced. He clinically resolved overtime with normal pituitary function and resolution of the pituitary mass. Currently, he continues on low-dose oral hydrocortisone at maintenance dosing and is stable from an oncological perspective.

Alternatively, our second patient was commenced on three days of i.v. methylprednisolone daily, followed by a tapering regimen of high-dose oral prednisolone for four weeks. She was also placed on $\mathrm{T}_{4}$ replacement therapy. Four weeks later, she improved clinically and radiological imaging demonstrated resolution of the pituitary tumour, hence an attempt to wean her steroid regimen was made. Her melanoma disease burden, however, progressed during this period and she ultimately lost her cancer battle within the following month.

It is important to note that our patients demonstrated symptomatic improvement at scheduled follow-up appointments at different times (i.e. 2 weeks vs 4 weeks) post-commencement of treatment, indicating that response to hormone replacement therapy may vary among patients.

\section{Discussion}

Ipilimumab is a MAB to cytotoxic T-lymphocyte-associated antigen 4 that inhibits tumour progression by enhancing immune-mediated destruction of malignant cells (1) (2) (3).

While this novel class of drugs hold promise in the treatment of various cancers, they also have the potential to unleash an autoimmune flare in susceptible patients, involving the gastrointestinal, endocrine and neurological systems (4). Recent studies have drawn attention to a group of endocrine immune-related adverse events, including $\mathrm{AH}$, thyroid disease, adrenal insufficiency and SIADH (2).

Observational studies suggest an association among dose and duration of therapy, clinical response and severity of the side effect profile. Multiple trials have used varying doses of ipilimumab ranging from $0.3-10 \mathrm{mg} / \mathrm{kg}$, with optimal clinical response shown to occur with $3 \mathrm{mg} / \mathrm{kg}$ (5). While affecting $<5 \%$ of all ipilimumab-treated patients, $\mathrm{AH}$ can cause life-threatening complications, particularly hypocortisolism, and tends to clinically manifest between administration of the third and fourth dose of infusions (6).

Fortunately, treatment involving high-dose steroids along with hormone replacement has shown promising results in terms of clinical, biochemical and radiographic resolution of the condition. Current studies report patient response to i.v. administration of high-dose steroids (5); however, our first case was treated successfully as an outpatient with oral prednisone. Further studies are needed to compare response to i.v. steroids vs oral steroids. Furthermore, MRI findings among $\mathrm{AH}$ patients have known to be quite varied, but typically involves diffuse enlargement of the pituitary gland, more often with hypointensity on T1-weighted imaging, heterogeneous enhancement and accompanied by thickening of the pituitary stalk (6) (7). Both cases demonstrated pituitary masses with hypointensity and heterogeneous changes, which responded very well to high-dose steroids indicating the inflammatory pathology.

A pituitary biopsy remains the gold standard in establishing the diagnosis and distinguishing hypophysitis from pituitary metastases. However, due to technical

A

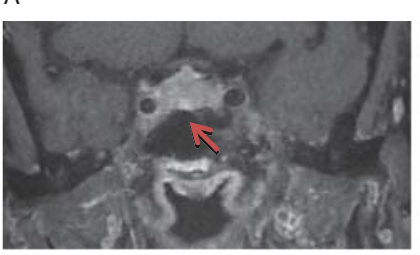

B

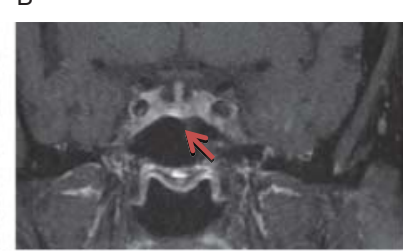

Figure 2

(A) Pre-treatment. Hypointense heterogeneous enhancement of the anterior pituitary gland, $12 \mathrm{~mm}$ craniocaudally and $13 \mathrm{~mm}$ antero-posteriorly on post-contrast T2 MRI head. (B) Post-treatment. Homogeneous enhancement of pituitary gland measuring $1 \times 0.3 \times 0.3 \mathrm{~cm}$ with central stalk completely clear of the optic chiasm. Arrows indicate pituitary masses. 
difficulties involved, this is not usually feasible. In such circumstances, clinical markers such as the acute presentation with hypocortisolism or hypopituitarism, normal prolactin levels, the hypointense lesion on imaging and absence of diabetes insipidus point towards hypophysitis rather than pituitary metastases from the melanoma. The therapeutic response to steroids confirms the diagnosis. Corsello et al. suggest that history of prior radiotherapy may have a protective role in preventing AH among patients treated with ipilimumab (8). This was not the case in Case 1, who was alone in receiving prior oncology treatment upon being commenced on ipilimumab. At this stage, predictive factors to identify those at risk of $\mathrm{AH}$ prior to therapy remain unclear, with neither of our patients having a known history of other autoimmune conditions, infections or allergies that could have potentially predisposed them to this immune-mediated reaction. Efficacy of ipilimumab recommencement post-treatment for $\mathrm{AH}$ is yet to be determined, with some studies predicting the same clinical response of AH to treatment in patients recommenced on the drug after hormone replacement therapy.

Most described cases seem to require ongoing steroid hormone replacement (8). In our cases, the first patient has been off $\mathrm{T}_{4}$ and on a maintenance dose of hydrocortisone, whereas, with our second case, we were unable to trial a taper of therapy due to deterioration in her clinical condition from the metastatic melanoma.

\section{Declaration of interest}

The authors declare that there is no conflict of interest that could be perceived as prejudicing the impartiality of the research reported.

\section{Funding}

This research did not receive any specific grant from any funding agency in the public, commercial or not-for-profit sector.
Patient consent

This is to confirm that patient consent was obtained from our study participant. Consent was not obtained from the second as she is deceased.

\section{References}

1 Yang JC, Hughes M, Kammula U, Royal R, Sherry RM, Topalian SL, Suri KB, Levy C, Allen T, Mavroukakis S et al. 2007 Ipilimumab (anti-CTLA4 antibody) causes regression of metastatic renal cell cancer associated with enteritis and hypophysitis. Journal of Immunotherapy 30 825-830. (doi:10.1097/CJI.0b013e318156e47e)

2 Torino F, Barnabei A, De Vecchis L, Salvatori R \& Corsello SM 2012 Hypophysitis induced by monoclonal antibodies to cytotoxic $\mathrm{T}$ lymphocyte antigen 4: challenges from a new cause of a rare disease. Oncologist 17 525-535. (doi:10.1634/theoncologist.2011-0404)

3 Torino F, Barnabei A, Paragliola RM, Marchetti P, Salvatori R \& Corsello SM 2013 Endocrine side-effects of anti-cancer drugs: mAbs and pituitary dysfunction: clinical evidence and pathogenic hypotheses. European Journal of Endocrinology 169 R153-R164. (doi:10.1530/EJE-13-0434)

4 Brahmer JR, Tykodi SS, Chow LQ, Hwu WJ, Topalian SL, Hwu P et al. 2012 Safety and activity of anti-PD-L1 antibody in patients with advanced cancer. New England Journal of Medicine 366 2455-2465. (doi:10.1056/NEJMoa1200694)

5 Downey SG, Klapper JA, Smith FO, Yang JC, Sherry RM, Royal RE, Kammula US, Hughes MS, Allen TE, Levy CL et al. 2007 Prognostic factors related to clinical response in patients with metastatic melanoma treated by CTL-associated antigen- 4 blockade. Clinical Cancer Research $\mathbf{1 3}$ 6681-6688. (doi:10.1158/1078-0432.CCR-07-0187)

6 Weber JS, Kahler KC \& Hauschild A 2012 Management of immunerelated adverse events and kinetics of response with ipilimumab. Journal of Clinical Oncology 30 2691-2697. (doi:10.1200/JCO.2012.41. 6750)

7 Juszczak A, Gupta A, Karavitaki N, Middleton MR \& Grossman AB 2012 Ipilimumab: a novel immunomodulating therapy causing autoimmune hypophysitis: a case report and review. European Journal of Endocrinology 167 1-5. (doi:10.1530/EJE-12-0167)

8 Carpenter KJ, Murtagh RD, Lilienfeld H, Weber J \& Murtagh FR 2009 Ipilimumab-induced hypophysitis: MR imaging findings. AJNR. American Journal of Neuroradiology 30 1751-1753. (doi:10.3174/ ajnr.A1623)

9 Corsello SM, Barnabei A, Marchetti P, De Vecchis L, Salvatori R \& Torino F 2013 Endocrine side effects induced by immune checkpoint inhibitors. Journal of Clinical Endocrinology and Metabolism 98 1361-1375. (doi:10.1210/jc.2012-4075)

Received in final form 16 November 2014

Accepted 21 November 2014 